\section{OPEN ACCESS}

Edited by:

Akio Adachi,

Tokushima University, Japan

Reviewed by:

Yashpal S. Malik,

Indian Veterinary Research Institute,

India

Joana Rocha-Pereira

KU Leuven, Belgium

Abimbola O. Kolawole,

University of Michigan Health System,

United States

*Correspondence:

Akira Nakanish

nakanish@ncgg.go.jp

Kazuhiko Katayama

katayama@lisci.kitasato-u.ac.jp

†These authors have contributed equally to this work.

Specialty section: This article was submitted to Virology,

a section of the journal

Frontiers in Microbiology

Received: 08 January 2017

Accepted: 30 May 2017

Published: 15 June 2017

Citation:

Kitamoto T, Takai-Todaka R, Kato A

Kanamori K, Takagi H, Yoshida K, Katayama K and Nakanishi A (2017)

Viral Population Changes during

Murine Norovirus Propagation in RAW

264.7 Cells. Front. Microbiol. 8:1091.

doi: 10.3389/fmicb.2017.01091

\title{
Viral Population Changes during Murine Norovirus Propagation in RAW 264.7 Cells
}

\author{
Takuya Kitamoto ${ }^{1 t}$, Reiko Takai-Todaka ${ }^{2+}$, Akiko Kato', Kumiko Kanamori \\ Hirotaka Takagi ${ }^{4}$, Kazuhiro Yoshida ${ }^{3}$, Kazuhiko Katayama ${ }^{2,5 *}$ and Akira Nakanishi ${ }^{1,3 *}$
}

\begin{abstract}
${ }^{1}$ Laboratory of Radiation Safety, National Center for Geriatrics and Gerontology, Obu, Japan, ${ }^{2}$ Laboratory of Gastroenteritis Viruses, Virology II, National Institute for Infectious Diseases, Musashimurayama, Japan, ${ }^{3}$ Section of Gene Therapy, Department of Aging Intervention, National Center for Geriatrics and Gerontology, Obu, Japan, ${ }^{4}$ Division of Biosafety Control and Research, National Institute for Infectious Diseases, Tokyo, Japan, ${ }^{5}$ Laboratory of Viral Infection I, Graduate School of Infection Control Sciences, Kitasato Institute for Life Sciences, Kitasato University, Tokyo, Japan
\end{abstract}

Laboratory adaptation of viruses is an essential technique for basic virology research, including the generation of attenuated vaccine strains, although the principles of cell adaptation remain largely unknown. Deep sequencing of murine norovirus (MuNoV) S7 during serial passages in RAW264.7 cells showed that the frequencies of viral variants were altered more dynamically than previously reported. Serial passages of the virus following two different multiplicity of infections gave rise to distinct haplotypes, implying that multiple cell-adaptable sequences were present in the founder population. Nucleotide variants lost during passage were assembled into a viral genome representative of that prior to cell adaptation, which was unable to generate viral particles upon infection in cultured cells. In addition, presence of the reconstructed genome interfered with production of infectious particles from viruses that were fully adapted to in vitro culture. Although the key nucleotide changes dictating cell adaptation of MuNoV S7 viral infection are yet to be elucidated, our results revealed the elaborate interplay among selected sequences of viral variants better adapted to propagation in cell culture. Such knowledge will be instrumental in understanding the processes necessary for the laboratory adaptation of viruses, especially to those without relevant cell culture systems.

Keywords: norovirus, RAW264.7 cells, cell adaptation, mouse, Calicivirus

\section{INTRODUCTION}

Virus propagation in vitro using cultured cells is essential for virology research and for the production of attenuated virus for use in vaccines, including those for measles, polio, and rabies (Minor, 2015); however, the processes shaping viral adaptation to cell culture conditions remain poorly understood.

Norovirus (NoV) is the most prevalent cause of viral gastroenteritis worldwide. The virus belongs to Caliciviridae family and consists of a single-stranded, positive-sense, $\sim 7.6-\mathrm{kb}$ RNA genome that includes a $3^{\prime}$ poly(A) tail. The genome harbors three open reading frames (ORFs) - ORF1 encodes a non-structural (NS) polyprotein that constitutes NS1-2, NS3, NS4, NS5, NS6, and NS7 proteins, whereas ORF2 and ORF3 encode the major and minor capsid proteins VP1 
and VP2, respectively. In murine $\mathrm{NoV}(\mathrm{MuNoV})$, the accessory viral protein VF1 is encoded by the ORF2 region via an alternative VP1 reading frame (McFadden et al., 2011).

Due to the lack of a permissive cell line, it is currently infeasible to propagate human $\mathrm{NoV}(\mathrm{HuNoV})$ using conventional in vitro culture systems (Duizer et al., 2004). Limited growth of $\mathrm{HuNoV}$ in immunodeficient mice and in human B-cell lines co-cultured with intestinal bacteria has been reported (Taube et al., 2013; Jones et al., 2014). Recent advances in reverse-genetics (Katayama et al., 2014) and development of an enteroid culture system using intestinal stem cells have proven effective for $\mathrm{HuNoV}$ propagation in vitro (Ettayebi et al., 2016); however, this culture system is impractical for general use owing to its high cost. As such, MuNoV is often used as a surrogate model virus to study NoV because of its ability to proliferate in RAW264.7 cell cultures, as well as murine dendritic cells (Wobus et al., 2004), and of its similarity to HuNoV being infectious agents to gastrointestinal tract. The prototypic MuNoV strain MNV-1 was originally discovered as a lethal agent in $R A G 2 / S T A T 1^{-/-}$mice (Karst et al., 2003), although MNV-1 infections in wild-type mice caused little pathological changes and were quickly cleared by the host immune system. In comparison, a more prevalent $\mathrm{MuNoV}$ stain, CR3, causes persistent infection in the gastrointestinal tract of laboratory mice and is mostly benign to the host, even in immunodeficient mice (Thackray et al., 2007). The MuNoV S7 strain, which is the closest relative to CR3, also appeared to induce no pathological effects (Kitajima et al., 2009); however, whether the strain can cause persistent infection in mice is not known.

Cell adaptation of MNV-1 is associated with attenuated viral pathogenicity in host animals (Wobus et al., 2004). For instance, the V11I and E296K mutations in NS4 and VP1, respectively, are associated with an inability to cause lethal infection in immunodeficient mice, although the potential for growth in RAW264.7 cells appeared unaltered or slightly enhanced (Wobus et al., 2004; Bailey et al., 2008). MNV-1 genomic alterations during cell passages have been welldocumented by Sanger sequencing (Wobus et al., 2004; Bailey et al., 2008) and partially by deep sequencing (Mauroy et al., 2016); however, a detailed analysis of these changes in other $\mathrm{MuNoV}$ strains and their effect on cell adaptation has yet to be determined.

Here, we report on the detailed examination of MuNoV S7 population changes during cell passage using deep sequencing at two different multiplicity of infections (MOIs). The frequencies of sequence variations were monitored at each passage and linkages analyzed by haplotype reconstruction. Additionally, nucleotide variants lost during cell passage were assembled and used to construct a single genome, which was then examined for its ability to generate infectious particles and possible interactions with genomic sequences fully adapted to in vitro propagation. These results revealed dynamic associations between viral population changes in response to cell culture conditions and the complex interplay between viral variants during the selection of sequences better suited for in vitro propagation.

\section{MATERIALS AND METHODS}

\section{Cells and Viruses}

RAW264.7 cells (ATCC TIB-71; American Type Culture Collection, Manassas, VA, United States) were cultured according to the manufacturer's instructions. The MuNoV S7 strain was kindly provided by Dr. Yukinobu Tohya (Nihon University, Tokyo, Japan) and grown in RAW264.7 cells. Viral preparations derived from cell cultures passaged two or three times were designated as $\mathrm{P} 2$ and $\mathrm{P} 3$ virus, respectively.

$\mathrm{MuNoV}$ infection titers were examined according to the $50 \%$ cell culture infective dose (CCID50) as previously described (Katayama et al., 2014). Viral RNA copy number was quantified by real-time reverse transcription polymerase chain reaction (RT-PCR) to approximate viral particle number. Briefly, viral RNA was extracted using QIAamp viral RNA mini kit (Qiagen, Hilden, Germany) and amplified with iScript One-Step RT-PCR kit using SYBR Green (Bio-Rad, Hercules, CA, United States) and the primers, MNV 6082-6108 FW and MNV 6272-6246 RV (Supplemental Table 1), with CFX96 Real-time PCR detection system (Bio-Rad). Fixed amounts of in vitro-transcribed MuNoV RNA served as a standard (see "Generation of Recombinant MuNoV").

The number of viral particles required to achieve one infectious event was assessed using the P13 virus prepared from RAW264.7 cells passaged 10 times after infection with P3 virus. Particle numbers and infectious titers were estimated by quantifying viral RNA copy number and the CCID50, respectively. In our preliminary assessments, $\sim 500$ viral RNA copies were equivalent to one CCID50 unit, although others have shown that the ratio of infectious particles to viral genomes was approximately 1:100 (Baert et al., 2008; Fischer et al., 2015). This five-fold difference could result from differences in quantification method, strain characteristics, or cell culture conditions.

\section{Sequence Determination of Viral RNA by Sanger Sequencing}

Extracted RNA from P2 and P3 MuNoV-containing culture supernatants was resuspended in AVE buffer (Qiagen) and used to generate cDNA in triplicate reactions with either SuperScript III reverse transcriptase (Thermo Fisher Scientific, Waltham, MA, United States) or ReverTra Ace (Toyobo, Osaka, Japan). The resulting cDNA was amplified in triplicate reactions using PrimeStar enzymes (Takara, Otsu, Japan) and five primer sets (Supplemental Table 2), each generating fragments that partially overlapped with the adjacent fragments; thus, nine samples were generated each genomic fragment. The amplified fragments were then separated by gel electrophoresis, isolated, and sequenced with the Big Dye ready reaction kit 3.1 (Thermo Fisher Scientific) and a 3130xl Genetic Analyzer (Thermo Fisher Scientific).

\section{Preparation of Viral RNA for Next-Generation Sequencing (NGS)}

Two different viral populations were generated by passaging the P2 virus in RAW264.7 cells grown in a 6-cm dish. The first was applied to cells at an MOI $>5$ and incubated for 
$\sim 24 \mathrm{~h}$, at which point many of the cells had died, although more extensive cell death was observed at later passages. Cell supernatant $(\sim 4 \mathrm{~mL})$ was cleared of cell debris by two successive 10 min centrifugations-at $700 \times g$ and then $12,000 \times g-$ at $4^{\circ} \mathrm{C}$. The virus was then concentrated by ultracentrifugation with a SW50.1 rotor (Beckman Coulter, Fullerton, CA, United States) at $45,000 \mathrm{rpm}$ for $2 \mathrm{~h}$ at $4^{\circ} \mathrm{C}$, and the resulting pellet was resuspended in $0.1 \mathrm{~mL}$ AVE buffer (Qiagen) for RNA extraction with the QIAamp viral RNA mini kit (Qiagen).

The other viral population was generated by infecting the cells at an MOI of 1 CCID50 ( $~ 500$ viral particles)/cell and the supernatant was collected at 48 -h post infection (hpi). Cell supernatant $(\sim 4 \mathrm{~mL})$ from each passage was concentrated as described above, the RNA extracted using Isogen II (Takara), and then subsequently resuspended in RNase-free water.

\section{Reverse Transcription, Double-Stranded DNA Synthesis, Library Preparation, and Deep Sequencing}

Viral RNA was used to synthesize cDNA with Superscript III reverse transcriptase (Thermo Fisher Scientific), which was then amplified using PrimeStar GXL enzymes (Takara) and two sets of primers, MNV-1S 14-32/MNV-7A 5361-5380 and MNV-4S 49895008/TX30SXN (Supplemental Table 1). The resulting fragments encompassed the proximal and distal halves of the viral genome, respectively, with an overlap of $>300$ nucleotides. The DNA fragments were gel-isolated and $1 \mathrm{ng}$ used to prepare a cDNA library with the Nextera XT DNA Sample Prep Kit (Illumina, San Diego, CA, United States) according to manufacturer's instructions. Briefly, DNA was fragmented and tagged by the Nextera XT transposome and then used as a template in a $50-\mu \mathrm{L}$, 12-cycle PCR. The amplified DNA was processed as outlined in the Nextera XT protocol and purified with AMPureXP beads (Beckman).

The quality of the purified DNA libraries was assessed on a MultiNA MCE-202 Bioanalyzer (Shimadzu Corporation, Kyoto, Japan). Nucleotide sequencing was performed on an Illumina MiSeq sequencer with a MiSeq Reagent Kit v2 (Illumina) to generate 151 paired-end reads.

\section{Data Processing and Analysis}

Short-reads were trimmed and mapped to the $\mathrm{MuNoV}$ reference sequence (GenBank: AB435515.1) using CLC Genomics Workbench 4.65 (CLC Bio, Cambridge, MA, United States) with default alignment settings. BAM files were exported from the CLC Genomics Workbench and analyzed using SAMtools v1.3.1 (Li et al., 2009) to extract sequence coverage and relevant statistics (Supplemental Presentation 1). Major nucleotide variants were called using CLC Genomics Workbench and their frequencies sampled and extracted using SAMtools (Supplemental Table 4). QuasiRecomb version 1.2 (Topfer et al., 2013) was used to reconstruct the viral haplotypes from sequencing data in BAM files. Local haplotype reconstruction across genes was performed using the default setting and conservative parameters to determine the inferred haplotypes and estimate their frequencies.

\section{Construction of DNA}

Unless noted, PrimeStar enzymes (Takara) and the InFusion system (Takara) were used for PCR-fragment generation and fragment insertion into plasmid DNA, respectively. Primers used in this study are listed in Supplemental Table 1. All construct sequences were confirmed by Sanger sequencing.

All genomic variations different from the MuNoV S7 cDNA reference sequence (GenBank: AB435515.1) or PP3 detected by deep sequencing (Supplemental Table 4) were assembled and synthesized as a single artificial genome-termed the PP2 composite sequence (PP2com). The nucleotide differences between PP2com and PP3 spanned from nucleotides 87 to 6752 in the viral genome. The EcoRI and BstBI sites positioned at -73 and 6996 bp in pMNV S7, respectively, (Katayama et al., 2014) were used to replace the original $\mathrm{MuNoV}$ sequence with that of PP2com. The $5^{\prime}$-proximal sequence up to the EcoRI site was also included in the synthesis.

pMNV ORF1 PP2, harboring the ORF1 from PP2com, was constructed by exchanging the PP3 sequence between the XhoI and BstBI restriction sites with the ORF2 and ORF3 from pMNV PP2, as well as a small region of the RNA-dependent RNA polymerase-coding sequence that included silent mutations at +4745 and +4877 bp in the genome.

Similarly, pMNV ORF23 PP2, harboring the ORF2 and ORF3 from PP2com, was generated by replacing the pMNV S7 fragment between the XhoI and BstBI restriction sites in with the PP2 sequence from pMNV PP2.

pT7 MNV S7 was generated from pMNV S7 (Katayama et al., 2014) by replacing the EF1-alpha promoter between the SspI and MluI restriction sites and with the T7-promoter sequence. First, two overlapping PCR fragments, A and B, were generated by PCR using pMNV S7 as the template with the primers, SspI FW and T7 MNV RV for A, and MluI RV and T7 MNV FW for B (Supplemental Table 1). The two fragments were then mixed and used as the templates for making insert by PCR using SspI FW and MluI RV as the primers for replacing SspI-MluI fragment of pMNV S7. The resultant pT7 MNVS7 harbors a truncated T7 promoter sequence ( $5^{\prime}$-TAATACGACTCACTATA- $\left.3^{\prime}\right)$ placed proximal to the MNV genomic cDNA sequence for generate RNA with $5^{\prime}$ ends identical to that of natural $\mathrm{MuNoV}$ upon in vitro transcription.

\section{Generation of Recombinant MuNoV}

Recombinant MuNoV was produced using a plasmid-based reverse-genetics system as described previously (Katayama et al., 2014). The pMNV S7, pMNV PP2, pMNV ORF1 PP2, and pMNV ORF23 PP2 plasmids were transfected individually or in combination into $293 \mathrm{~T}$ cells cultured in $35-\mathrm{mm}$ dishes by mixing $4 \mu \mathrm{g}$ DNA, $8 \mu \mathrm{L}$ P3000 reagent, and $12 \mu \mathrm{L}$ Lipofectamine 3000 (Thermo Fisher Scientific) in $400 \mu \mathrm{L}$ Opti-MEM (Thermo Fisher Scientific). Supernatants from transfected cultures were collected 48 -h post-transfection and used to infect RAW264.7 cells. The transfected cells were also collected to confirm viral protein expression by Western blotting. 
In addition, recombinant $\mathrm{MuNoV}$ was also generated using a RNA-based reverse-genetics system as previously described (Arias et al., 2012). Briefly, a series of pT7 MNV constructs was linearized with AscI and used as the template for in vitro transcription with the T7 RiboMax express large-scale RNAproduction system (Promega, Fitchburg, WI, United States). The synthesized RNA was purified using a Megaclear kit (Thermo Fisher Scientific) followed by $5^{\prime}$-end capping by ScriptCap (Illumina). The capped RNA was then re-purified and used to transfect $293 \mathrm{~T}$ cells in $35-\mathrm{mm}$ dishes with $4 \mu \mathrm{g}$ RNA and $8 \mu \mathrm{L}$ Lipofectamine 2000 (Thermo Fisher Scientific). The viral supernatant was collected $24 \mathrm{~h}$ later and used to infect RAW 264.7 cells. The virus was passaged once in RAW264.7 cells to obtain a sufficient titer for cell-infection experiments.

\section{Western Blotting}

Viral proteins from transfected 293T cells were analyzed by Western blotting as previously described (Haga et al., 2016). Briefly, the cell pellet was resuspended in calcium- and magnesium-free Dulbecco's Phosphate-Buffered Saline $\left(\mathrm{PBS}^{-}\right)$, disrupted by sonication, and centrifuged to remove cell debris. The protein in each sample was quantified and then denatured with SDS-dye buffer (Wako Chemicals, Tokyo, Japan). Aliquots containing about $\sim 10 \mu \mathrm{g}$ protein were loaded into each lane, separated by 5-20\% SDS-PAGE, and transferred onto a polyvinylidene fluoride (PVDF) membrane using a Trans-Blot system (Bio-Rad). The membrane was blocked with solution containing 2.5\% skim milk and $0.5 \times$ PVDF blocking buffer (Toyobo), followed by rabbit anti-NS1-2 in solution 1 from the Can Get Signal Kit (Toyobo). After washing, immunoreactive bands were detected with horseradish peroxidase (HRP)-conjugated anti-rabbit IgG antibody in solution 2. Chemiluminescence was detected by ImmunoStar (Wako Chemicals) and recorded using a LAS4000 (GE Healthcare, Little Chalfont, United Kingdom). The membrane was then stripped with Restore Plus Western blot stripping buffer (Thermo Fisher Scientific) and reprobed with HRPconjugated guinea pig anti-NS7 and mouse anti-actin (Wako Chemicals).

\section{Immunofluorescent Staining}

Supernatants $(\sim 0.5 \mathrm{~mL})$ from cells transfected with $\mathrm{MuNoV}$ genome constructs were used to infect RAW264.7 cells grown on coverslips in each well of a 12-well plate. The cells were fixed $48 \mathrm{~h}$ later with $4 \%$ paraformaldehyde $(\mathrm{pH} 7.5)$ and examined for viral protein expression by immunocytochemistry. Mixtures of primary anti-sera, guinea pig anti-VPg, and rabbit anti-VP1 in PBS $^{-}$supplemented with $0.5 \%$ Triton X-100 were used to detect the respective proteins, followed by AlexaFluor 488-conjugated anti-guinea pig IgG and AlexaFluor555-conjugated antirabbit IgG secondary antibodies. Samples were embedded in Vectashield mounting medium (Vector Labs., Burlingame, CA, United States) containing 4',6-diamidino-2-phenylindole (DAPI) and imaged with an epifluorescent microscope (BZ9000; Keyence, Osaka, Japan).

\section{RESULTS}

The MuNoV S7 PP3 sequence (GenBank: AB435515.1) originated from a molecular clone obtained from the P3 virus that was passaged three times in RAW264.7 cells (Figure 1). The recombinant virus generated from the PP3 sequence by the plasmid-based reverse-genetics system grew well in RAW264.7 cells (Katayama et al., 2014); however, Sanger sequencing revealed that the P3 "consensus" sequence differed by more than 55 nucleotides with 11 amino acid-changes from the PP3 sequence (Supplemental Table 3). Similarly the consensus sequence of P2 virus, which was passaged twice in RAW264.7 cells, differed by 48 nucleotides with 11 missense changes from the reference sequence suggesting that both viral preparations contained pools of heterogeneous sequences (Supplemental Table 3). After 10 passages of the P3 virus in RAW264.7 cells (P13 virus), the consensus viral sequence became identical to that of PP3, thereby confirming that this MuNoV S7 sequence was representative of the one adapted to growth in RAW264.7 cells.

Because earlier passages of MuNoV S7 isolates-including the P2 virus-contained variable clones differing from those of PP3, this suggested that the original viral isolate contained multiple variants, which were further refined by continued propagation in RAW264.7 cells. To further delineate the process by which this occurred, viral sequences from each passage were examined by deep sequencing. For this, viral RNA was prepared from the two set of passages inoculated at different MOIs. The first culture was passaged at an MOI > $5 \mathrm{CCID50/cell,} \mathrm{assuming}$ that multiple viral clones would likely coexist in a single cell upon inoculation (high-MOI passage). After six passages, viral RNA was extracted from the infected culture supernatants for sequencing and defined as $\mathrm{H} 1$ to $\mathrm{H} 6$ for passages one to six. The second culture was inoculated at an MOI of 1 CCID50/cell, assuming that $\sim 63 \%$ of cells were infected and $37 \%$ of those were infected with only one virus (a low-MOI passage). It should also be noted that MOIs were only valid at inoculation, as much higher MOI would be expected after single round of viral propagation and release at subsequent infection to adjacent cells. Culture supernatants were harvested over nine passages. Samples L1 to L4 represented those collected from passages two to five, whereas L5, L6, and L7 were from passages six, seven, and nine, respectively.

Deep sequencing of the viral RNA samples H1-H6 and L1-L7 revealed dynamic sequence changes in the viral population. Highand low-MOI passages showed a total of 89 and 103 nucleotide differences from the PP3 sequence, respectively, of which 87 were shared. The variations included 17 non-synonymous and 22 missense mutations, including 8 shared among the passages (Figure 2 and Supplemental Table 4). The proportion of nonPP3 sequences-referred to as PP2 sequences-in each passage is summarized in Figure 2, with detailed data provided in Supplemental Table 4. Overall changes in nucleotide frequencies in the low-MOI passage were far more evident than those observed with the high-MOI infection, of which many in lowMOI Passage 9 (sample L7) reached nearly zero, indicating that several variants were lost. In contrast, variant frequencies in 


\section{A}
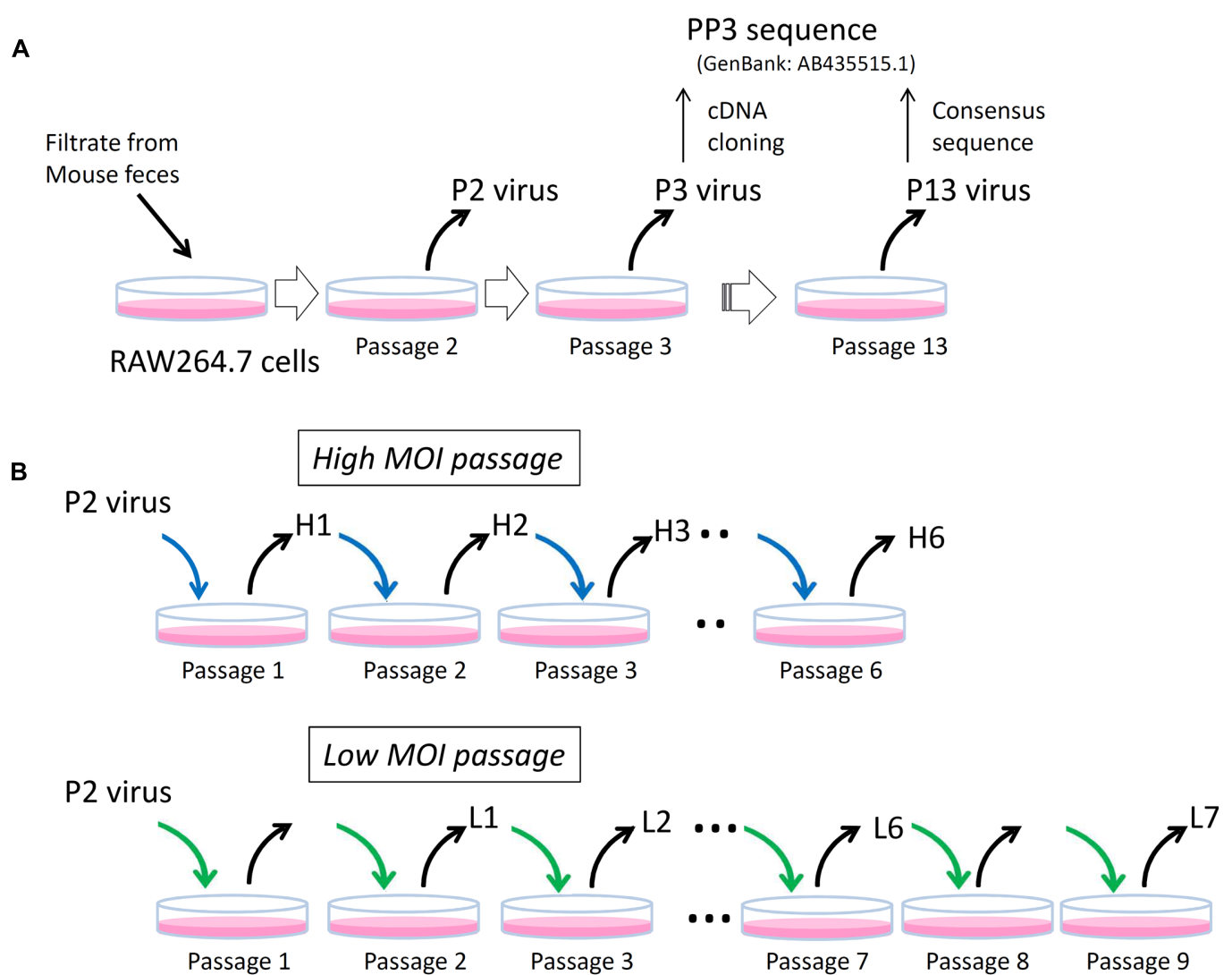

FIGURE 1 | Schematics of experimental layout. (A) P2, P3, and P13 viruses. The P2 and P3 viruses are MuNoV preparations passaged in RAW264.7 cells twice (Passage 2) and three times (Passage 3), respectively. Representative molecular clone of MuNoV S7, PP3, was generated by cDNA cloning from P3 virus. After ten passages of P3 virus (Passage 13), which designated as P13 virus, the consensus sequence of the virus became identical to that of PP3. (B) High and low MOI passages. Two different $\mathrm{MOI}$ conditions were used to inoculate P2 virus and subsequent passages in RAW264.7 cells. The blue and green arrows indicate viral inoculation under $\mathrm{MOI}>5 \mathrm{CCID50}$ unit and $\mathrm{MOI}=1 \mathrm{CCID50}$ unit, respectively. Note in "Low MOI passage" number of passages and the samples do not match; no viral samples were taken at passages 1 and 8.

high-MOI samples showed relatively smaller changes. Moreover, further examination of the low-MOI variants revealed temporal shifts in some nucleotide frequencies (Supplemental Table 4).

To investigate nucleotide frequency linkages, we used QuasiRecomb to generate haplotypes (Topfer et al., 2013). QuasiRecomb implements a hidden Markov model to infer viral quasispecies from deep-coverage NGS data using an expectation-maximization algorithm for maximum posteriorparameter estimation and explicitly accounts for paired-end information. Haplotype reconstructions for the full genome, ORF1, ORF2, and ORF3 are shown in Table 1A. Computational processing required deep reads and $>1000$ coverages were prerequisite for distinguishing single-nucleotide polymorphisms and misreads by deep sequencing (Topfer et al., 2013). Not surprisingly, applying this method to samples H1, L3, and L4 with $<1000$ coverages on average resulted in large numbers of haplotypes, which likely included misreads. Similar findings were observed with $\mathrm{H} 3$ and $\mathrm{H} 4$, although it was unknown whether this was attributed to the relatively smaller degree of coverage. Results of comparisons from the remaining samples $(\mathrm{H} 2, \mathrm{H} 5$, and H6 from high-MOI passage and L1 and L4-L7 from low-MOI passage) revealed a general trend of fewer identified haplotypes in low-MOI cultures. Moreover, the ORF1 region generated one or two haplotypes during the final passages of both MOIs, whereas the ORF2 region showed 15 and 2 haplotypes for high- and lowMOI cultures, respectively, which could explain the differences in haplotype number observed for the full genomes (29 and 2 for high- and low-MOI passages, respectively).

While the conservative settings only reconstruct major haplotypes and disregard minor ones, the default setting includes minor haplotypes and generates details for changes in haplotype frequencies. However, haplotype reconstructions for ORF1 and the full genome were unsuccessful with the default settings because of inadequate coverage. As such, we only reconstructed the haplotypes with the default setting for ORF2 and ORF3 regions (Table 1B). Notably, the ORF2 region harbored a large number of viral haplotypes; 3569 and 5343 in $\mathrm{H} 1$ and L1, respectively. In addition, fewer haplotypes were observed in late passages from both MOIs, indicative of haplotype convergence, although more haplotypes were present in $\mathrm{H} 6$ as compared to L7. In particular, major haplotype frequencies associated with the ORF2 region observed were 0.691 and 0.059 in $\mathrm{H} 6$ and 

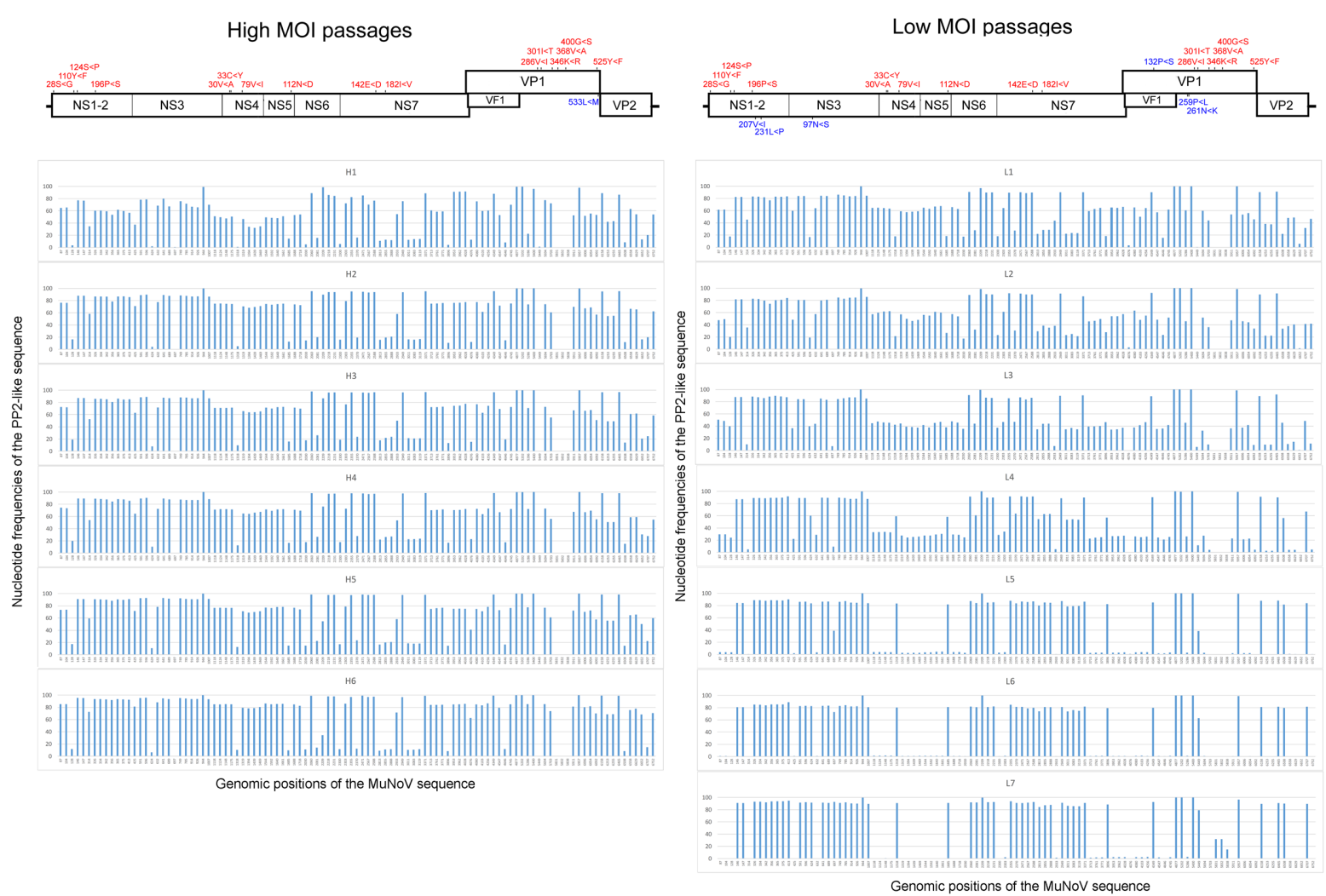

FIGURE 2 | Frequencies of nucleotide differences between the PP2 and PP3 sequences during MuNoV cell passage. Changes in PP2-sequence frequencies during high- and low-MOI passages (Left and Right, respectively). The horizontal axis indicates the genomic position of the sequences, and the vertical axis indicates the nucleotide frequencies of the PP2-like sequence. Upper panels depict schematics of the MuNoV genome, with indications of non-synonymous nucleotide changes translated to amino acids. Numbers indicate amino acid position affected by the nucleotide variations, and capital letters flanking " $<$ " represent amino acids encoded by PP3 or PP2 sequences, respectively. Letters in red are common changes observed between the high- and low-MOI passages, and those in blue represent changes unique between passages.

0.899 and 0.334 for L7 using the conservative and default settings, respectively (Table 1). For the ORF3 region, haplotype reconstruction using the conservative settings generated single major haplotypes for the final passages at both MOIs and major haplotype frequencies of 0.688 and 0.924 for the high- and lowMOIs using the default setting (Table 1).

The major haplotypes identified in the full genome sequences from the final high- and low-MOI passages were distinctwith 81 and 52 nucleotide differences from the original PP3 sequence, respectively, and 35 of which were shared (Figure 3). Those changes consisted of 16 non-synonymous and 13 missense mutations, including 8 shared (Figure 3) and did not seem localized to a particular region in the viral genome. Notably, the major haplotype sequences from the final passages generated from the full genome and individual ORFs were identical. Moreover, the major haplotypes for ORF2 and ORF3 in the final passages generated by default and conservative settings were identical.

Close examination of major ORF2 haplotypes in low MOI passage with the default settings revealed dynamic changes in number and frequency. As such, we examined the frequencies of 15 haplotypes that accounted for $>3 \%$ of those observed in any single passage and found temporal shifts for several haplotypes that were distinct from the majority haplotypes in the final passage (Figure 4A). Similar changes in ORF3 haplotype frequencies were also noted (Figure 4B).

Genetic variants present in the majority of the viral population in the final passages represented those better adapted for propagation in RAW264.7 cells. Conversely, variants present in earlier passages that were not observed in later passages were likely unfit for growth in RAW264.7 cells. Therefore, viruses harboring PP2-like sequences may represent viral populations not adapted to in vitro propagation. We cloned the PP2-like sequences from viral RNA extracted from the PP3 virus and generated recombinant viruses; however, the cloned sequences only covered a minor fraction of the PP2 nucleotide variations, and the recombinant viruses showed no growth defects in RAW264.7 cells (Supplemental Presentation 2).

To determine the cumulative effect of all PP2 nucleotide variations on viral propagation in RAW264.7 cells, we synthesized a PP2 genome that incorporated all nucleotide variations that differed from PP3 detected by deep sequencing (Supplemental Table 4). The PP2com genome was inserted into a PP3 construct previously shown to produce infectious particles 
TABLE 1 | Haplotype frequencies generated by QuasiRecomb.

(A)

\begin{tabular}{|c|c|c|c|c|c|c|c|c|c|c|c|c|c|}
\hline & \multicolumn{6}{|c|}{ High-MOI passages } & \multicolumn{7}{|c|}{ Low-MOI passages } \\
\hline & H1 & $\mathrm{H} 2$ & H3 & H4 & H5 & H6 & L1 & L2 & L3 & L4 & L5 & L6 & L7 \\
\hline \multicolumn{14}{|l|}{ Full genome } \\
\hline Average coverage & 847 & 5975 & 2380 & 2058 & 3792 & 2883 & 3056 & 223 & 227 & 2295 & 2506 & 4032 & 3148 \\
\hline No. of haplotypes & 9966 & 16 & 2903 & 4176 & 12 & 29 & 34 & 9740 & 8575 & 87 & 1 & 8 & 2 \\
\hline Freq. $>3 \%$ & 0 & 6 & 0 & 0 & 4 & 5 & 7 & 0 & 0 & 12 & 1 & 2 & 2 \\
\hline Freq. (1st) & 0.0003 & 0.317 & 0.009 & 0.008 & 0.313 & 0.54 & 0.464 & 0.004 & 0.001 & 0.258 & 1 & 0.923 & 0.908 \\
\hline \multicolumn{14}{|l|}{ ORF1 } \\
\hline Average coverage & 871 & 6018 & 2464 & 2098 & 3829 & 2950 & 3266 & 234 & 240 & 2382 & 2636 & 4233 & 3153 \\
\hline No. of haplotypes & 8561 & 8 & 170 & 611 & 10 & 2 & 20 & 5973 & 5026 & 54 & 1 & 2 & 1 \\
\hline Freq. $>3 \%$ & 0 & 7 & 5 & 6 & 6 & 2 & 7 & 0 & 0 & 2 & 1 & 2 & 1 \\
\hline Freq. (1st) & 0.001 & 0.438 & 0.424 & 0.527 & 0.446 & 0.787 & 0.372 & 0.002 & 0.003 & 0.681 & 1 & 0.715 & 1 \\
\hline \multicolumn{14}{|l|}{ ORF2 } \\
\hline Average coverage & 870 & 6407 & 2450 & 2183 & 4073 & 3093 & 2772 & 201 & 201 & 2118 & 2303 & 3728 & 3217 \\
\hline No. of haplotypes & 64 & 4 & 215 & 222 & 4 & 15 & 501 & 124 & 8 & 1 & 1 & 1 & 2 \\
\hline Freq. $>3 \%$ & 6 & 2 & 11 & 7 & 2 & 5 & 0 & 10 & 7 & 1 & 1 & 1 & 2 \\
\hline Freq. (1st) & 0.044 & 0.65 & 0.06 & 0.051 & 0.658 & 0.691 & 0.023 & 0.067 & 0.379 & 1 & 1 & 1 & 0.899 \\
\hline \multicolumn{14}{|l|}{ ORF3 } \\
\hline Average coverage & 716 & 5189 & 1780 & 1643 & 3194 & 2131 & 2434 & 218 & 215 & 2284 & 2269 & 3662 & 3243 \\
\hline No. of haplotypes & 1 & 1 & 1 & 1 & 1 & 1 & 1 & 1 & 2 & 1 & 1 & 1 & 1 \\
\hline Freq. $>3 \%$ & 1 & 1 & 1 & 1 & 1 & 1 & 1 & 1 & 2 & 1 & 1 & 1 & 1 \\
\hline Freq. (1st) & 1 & 1 & 1 & 1 & 1 & 1 & 1 & 1 & 0.556 & 1 & 1 & 1 & 1 \\
\hline
\end{tabular}

(B)

\begin{tabular}{|c|c|c|c|c|c|c|c|c|c|c|c|c|c|}
\hline & \multicolumn{6}{|c|}{ High-MOI passages } & \multicolumn{7}{|c|}{ Low-MOI passages } \\
\hline & H1 & $\mathrm{H} 2$ & H3 & H4 & H5 & H6 & L1 & L2 & L3 & L4 & L5 & L6 & L7 \\
\hline \multicolumn{14}{|l|}{ ORF2 } \\
\hline Average coverage & 870 & 6407 & 2450 & 2183 & 4073 & 3093 & 2,772 & 201 & 201 & 2,118 & 2,303 & 3,728 & 3,217 \\
\hline No. of haplotypes & 3569 & 3382 & 4118 & 4455 & 3978 & 1623 & 5343 & 3976 & 1876 & 972 & 131 & 88 & 117 \\
\hline Freq. $>3 \%$ & 0 & 0 & 0 & 0 & 0 & 1 & 0 & 0 & 0 & 4 & 6 & 7 & 6 \\
\hline Freq. (1st) & 0.004 & 0.008 & 0.004 & 0.004 & 0.005 & 0.059 & 0.002 & 0.003 & 0.023 & 0.101 & 0.377 & 0.364 & 0.334 \\
\hline \multicolumn{14}{|l|}{ ORF3 } \\
\hline Average coverage & 716 & 5189 & 1780 & 1643 & 3194 & 2131 & 2434 & 218 & 215 & 2284 & 2269 & 3662 & 3243 \\
\hline No. of haplotypes & 4 & 4 & 4 & 4 & 4 & 4 & 8 & 4 & 4 & 4 & 4 & 4 & 2 \\
\hline Freq. $>3 \%$ & 4 & 4 & 4 & 4 & 4 & 3 & 4 & 4 & 2 & 3 & 3 & 3 & 2 \\
\hline Freq. (1st) & 0.486 & 0.555 & 0.49 & 0.421 & 0.509 & 0.688 & 0.369 & 0.369 & 0.5 & 0.67 & 0.82 & 0.738 & 0.924 \\
\hline
\end{tabular}

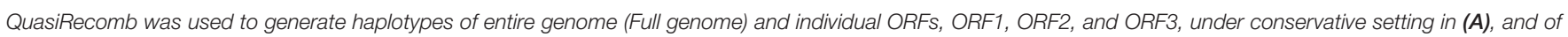

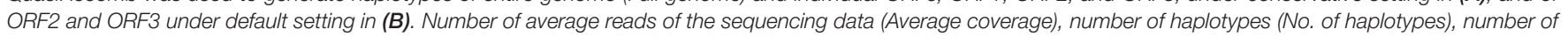
haplotypes accounted higher than 3\% (Freq. >3\%), and frequency of the major haplotype (Freq. 1st) from each passage are shown.

upon transfection into $293 \mathrm{~T}$ cells. Notably, the PP2com viral genome failed to produce infectious particles (Figure $5 \mathbf{B}, \mathbf{b}$ ), whereas the PP3 sequence generated high viral titers infectious to RAW264.7 cells (Figure 5B,a). Further, conversion of the PP2com ORF1 or ORF23 region to the PP3 sequence was unable to restore particle production (Figures $\mathbf{5 B}, \mathbf{c}, \mathbf{d}$ ). Subsequent analysis of ORF1 protein expression revealed that NS1-2 and NS7 were detectable in cells transfected with the PP2com sequence (Figure 5C); however, VP1 expression was undetectable, given that the extent of sub-genomic replication is low when using the plasmid-based reverse-genetics system
(Katayama et al., 2014). Examination of interactions between the PP2com and PP3 sequences showed that the PP2com sequence interfered with the production of infectious particles from the PP3 sequence, as co-transfection of the PP3 and PP2com genomes yielded no infection (Figure 5B,e). However, presence of the PP2com ORF1 region alone was insufficient to block the PP3-mediated virus production in one experiment (Figure 5B,f), but in two other experiments infectious virus was not produced from PP3 genome with the presence of ORF1 PP2 genome. In contrast, co-transfection of the PP3 genome and ORF23 PP2 generated infectious particles (Figure 5B,g), as did that of 

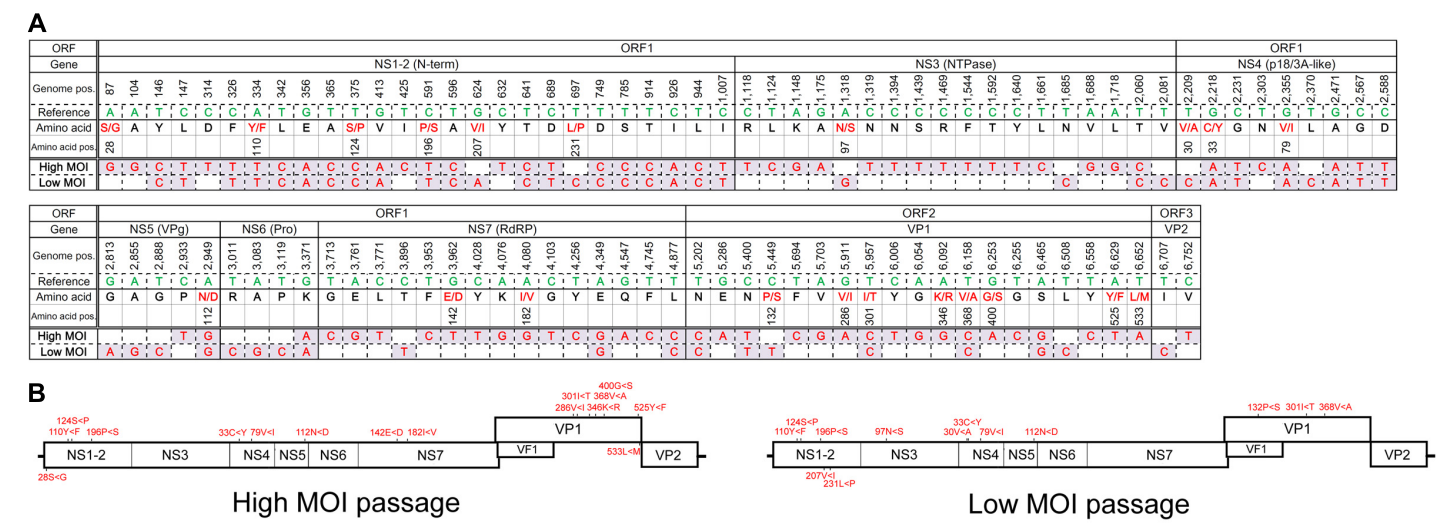

FIGURE 3 | Major haplotype sequences found following the final high- or low-MOI passage. (A) Sequence of the major haplotype following the final high- and low-MOI passages. Nucleotides that differed from those found in the PP3 (Reference) sequence are shown along with their genome position (Genome pos.) and the encoded amino acid residue (Amino acid) and their position (Amino acid pos.) in the PP3- or PP2-encoded protein, respectively. Non-synonymous changes are depicted in red. (B) Non-synonymous changes of each major haplotype are depicted in the MuNoV genome. Amino acid changes are described similar to those in Figure 2 .
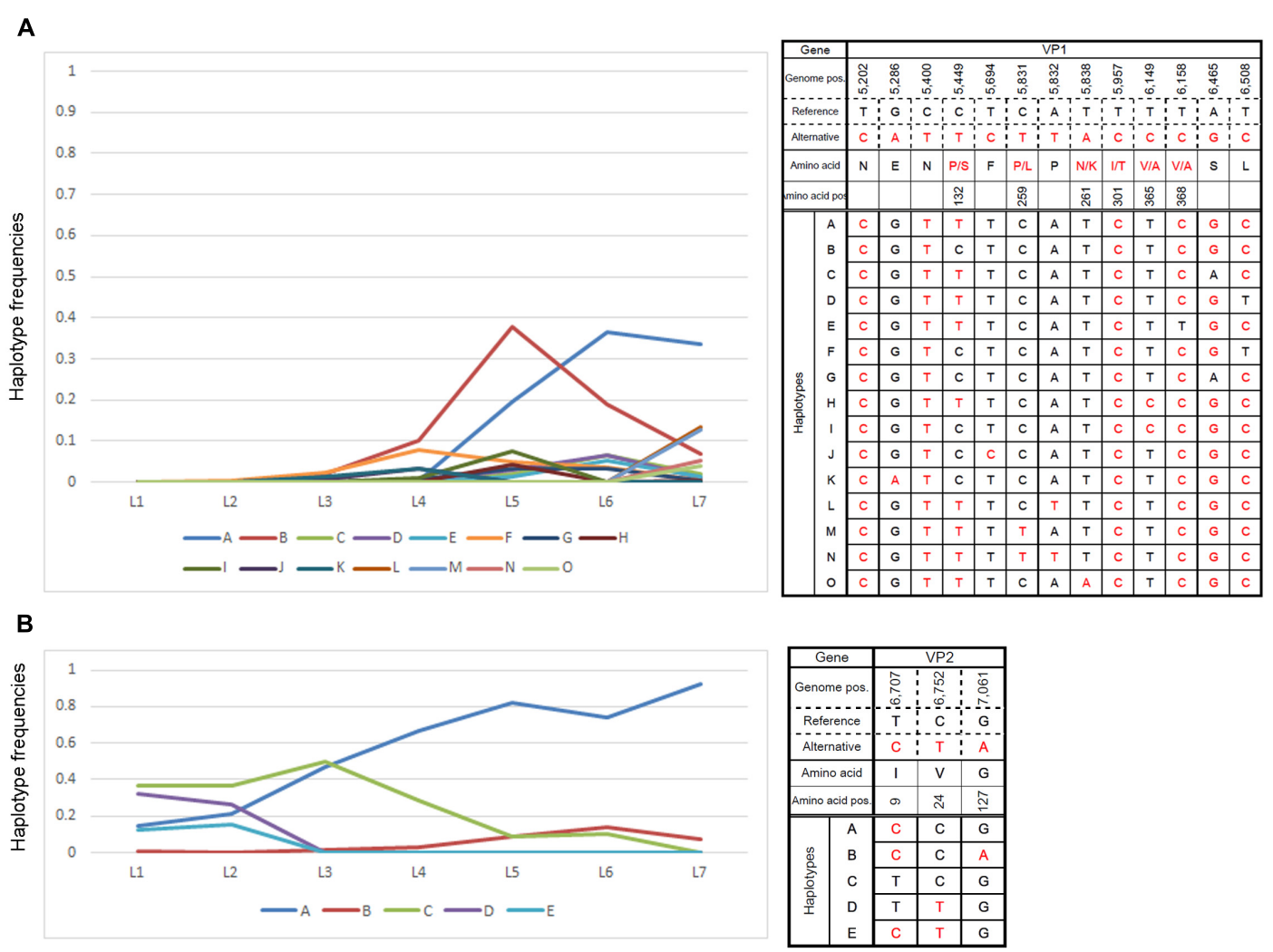

FIGURE 4 | Changes in major haplotype frequencies during each passage. Changes in haplotype frequencies in (A) VP1- and (B) VP2-encoding regions during low-MOI passages were examined by QuasiRecomb using the default setting. Fifteen haplotypes accounted for $>3 \%$ of the changes observed in any of the passages were selected and monitored for the frequency of nucleotide changes observed during each passage. The right panels indicate the haplotype sequence along with genome position and possible amino acid change. The left panels show changes in nucleotide frequencies of the selected haplotypes as a line graph. The vertical and horizontal axes indicate the haplotype frequencies and samples for the respective cell passage, respectively.

ORF1 PP3 and ORF23 PP2 (Figure 5B,h). The quantification of viral particles in the supernatant of cells transfected with PP3, PP3 + ORF23 PP2, and ORF1 PP2 + ORF23 PP2 contained approximately $2.45 \times 10^{3} / \mathrm{mL}, 2.67 \times 10^{4} / \mathrm{mL}$, and $8.09 \times 10^{3} / \mathrm{mL}$ CCID50, respectively, whereas no particles were found in supernatants from cells transfected with PP3 and ORF1 

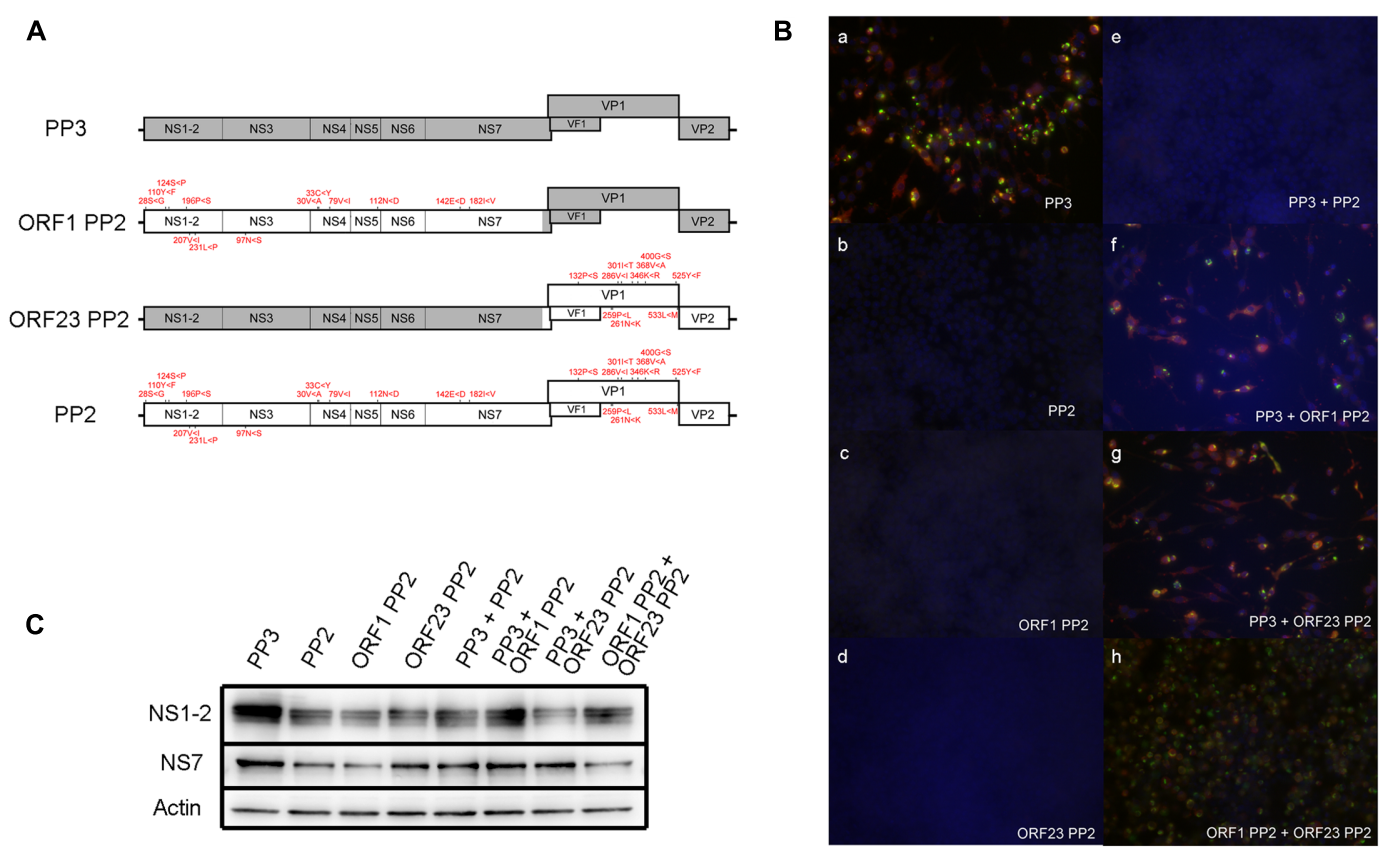

FIGURE 5 | Examination of the ability of the PP2com genome to generate infectious particles. (A) Schematic of the recombinant MuNoV genome depicting regions containing PP3 and PP2com sequences. The amino acid residues encoded by the PP2com genome that differed from those encoded by the PP3 genome are indicated in red. (B) Supernatant of transfected cells in (C) was used to infect RAW264.7 cells. At 48 hpi, cells were fixed and detected for VPg (Green) and VP1 (red) by immunofluorescent detection with staining for DNA by DAPI (Blue) (see "Immunofluorescent Staining" in Materials and Methods). (C) Detection of the ORF1 products NS1-2, NS7, and actin by Western blot from the lysates of 293T cells transfected with pMNVs expressing the respective genome. The first four sets of samples were prepared by cotransfecting $2 \mu \mathrm{g}$ of pT7 MNV S7 and $2 \mu \mathrm{g}$ each of plasmid expressing the designated recombinant MuNoV genome. The last four sets of the samples were prepared by transfecting the plasmid in combination with those expressing the respective genome.

PP2 cDNA. Collectively, these results indicated that the presence of PP2-like nucleotide variations attenuated viral growth in RAW264.7 cells and interfered with the production of infectious particles from fully adapted sequences. Moreover, variants in the ORF1 region exhibited a trans-dominant inhibitory effect; however, co-expression of ORF1 PP2 and ORF23 PP2 to $293 \mathrm{~T}$ cells generated particles infectious to RAW264.7 cells, indicating that interaction between the PP2com and PP3 sequence was complex-showing both complementation and interference-and dependent upon the context of the sequences.

\section{DISCUSSION}

In this study, we examined details associated with changes in nucleotide frequencies in the MuNoV genome during viral passage following infection with two different MOIs. Interestingly, the number of variants decreased over time depending on the culture conditions, with greater variations generally observed with high-MOI infection. Haplotype analysis revealed that the major haplotype sequences in the final passages differed between high- and low-MOI cultures, suggesting that the initial viral populations contained multiple cell-adaptable sequences. Moreover, the genetic variations lost during in-cell propagation were assembled into a single genome and used to transfect cells, but was unable to produce infectious particles itself, as well as those from cell-adapted PP3. Although the key nucleotide changes dictating cell adaptation in the MuNoV S7 strain have yet to be elucidated and will be examined in future work, our results revealed the elaborate interplay among viral variants to select the sequences better-adapted to propagation in cell culture. To elaborate, our experimental procedures are depicted in Figure 1.

Murine norovirus sequences among the known strains are relatively less divergent with mostly fixed genome lengths (Thackray et al., 2007); however, variations in the MuNoV S7 strain exhibit large diversity, indicating the coexistence of multiple haplotypes in the viral isolate. When compared with known nucleotide variations in the MNV-1 (Wobus et al., 2004) and MNV NIH2409 strains (Barron et al., 2011) during cell passage, only two synonymous variations at +1409 and +2081 in MNV-1 were found in the S7 strain (Wobus et al., 2004; Bailey et al., 2008), although no changes associated with enhanced viral growth were detected in this study. Instead, we discovered nearly 100 nucleotide variations-including $\sim 20$ nonsynonymous changes, the frequency of which dynamically altered during the viral passages. The nucleotide changes seemed evenly distributed throughout the genome with slight emphasis at the anterior coding region of ORF1 and very few in ORF3 and 3'UTR regions, which contrasted to the results by others reporting high degree of variation at the ORF3 region (Mauroy et al., 2016). 
Given the limited viability of the PP2com genome (Figure 5), these findings indicated that the processes associated with cell adaptation involved multiple rounds of nucleotide alteration or selection.

In this study, we selected viral passages from cells infected at two different MOIs. High-MOI passage might allow for the maintenance of sequence variations and avoid sequence convergence or opportunistic genetic drift that result from genetic bottlenecks because of small subsets in the viral population (Andino and Domingo, 2015). Even under such conditions, haplotype analysis indicated that a single haplotype could flourish to predominate the viral population (Figure 3), suggesting the presence of selective pressure for variants better suited for propagation in culture. Not surprisingly, low-MOI passage assumed conditions that resembled a genetic bottleneck wherein the majority of cells do not assume infection with multiple viral clones. Although the secondary infection over the following $48 \mathrm{~h}$ period did not warrant conditions limiting entry of infectious clones, sequences exhibited convergence during early phase passages (Figure 3) and showed changes in haplotype frequency indicative of genetic drift (Figure 4). Genetic drift depends on the diversity in the founder viral population and the number of host cells. Since approximately $1 \times 10^{6}$ cells were used in the present study, the viral populations selected during passage would be no less than $1 \times 10^{6}$. Thus, our observation of viral sequence convergence to a single haplotype likely resulted from selective pressure rather than genetic drift, where sequence selection occurs by chance.

The two different passages generated distinct haplotype sequences that predominated the final viral populations. It is possible that the major haplotypes observed in the low-MOI passage were the product of genetic drift and could differ with prolonged passage. Considering that two distinct haplotypes were observed during the two different passages, and since serial passage of the P3 virus generated a major haplotype identical to $\mathrm{PP} 3$, our results suggested the presence of multiple viral haplotypes in the original viral isolate or founder population.

Assuming that PP3 was among the most cell-adapted sequences, we constructed the PP2com genome using sequence variations lost during cell passage. As expected, the PP2com genome was unable to produce infectious particles upon expression using the reverse-genetic system (Figure 5). This was not attributed to defects in viral gene expression, as the protein products encoded by ORF1 were expressed at levels comparable with those of PP3. Moreover, the co-transfection of constructs expressing ORF1 PP2 and ORF23 PP2 generated viral particles infectious to RAW264.7 cells (Figures 5B,C). Interestingly, co-expression of the PP2com and PP3 genomes blocked the production of infectious particles from PP3, indicating the presence of sequence elements in PP2com that interfered with viral growth in trans. Further, presence of the PP2com ORF1 region blocked viral particle production from PP3 genome, but the PP2com ORF23 region only showed a marginal blockade (Figure 5B,g). Such effect could be an accumulative since ORF1 and ORF23 from PP2com harbored 81 and 24 nucleotide differences from PP3 sequence, respectively, thus the number of nucleotide differences could be positively correlated with the strength of interference. Such genetic interference could also depend upon the sequence context as co-expression of the ORF1 and ORF23 PP2 genomes generated infectious particles despite that the PP2com-derived ORF1 sequence was present (Figure 5B,h). In addition, the coexistence of multiple viral haplotypes has been shown to generate complex interplay, including interference irrespective of coding or non-coding sequences (Ojosnegros et al., 2010; Acevedo et al., 2014). Thus, presence of PP2com-like sequences in the founder viral population would likely impart negative effects on viral propagation in vitro.

Here, we used deep sequencing to reveal that the frequencies of MuNoV S7 variants dynamically changed to converge to a cell-adaptable viral sequence during multiple viral passages under cell-culture conditions. The selective process associated with passages of MuNoV S7 in the cultured cells was more complex than previously reported in MNV-1, where only two nucleotide changes were sufficient (Wobus et al., 2004; Bailey et al., 2008). Our findings that the artificial PP2com genome exhibited transdominant negative effects on viral growth against a cell-adapted genome suggested elaborate interplay among viral variants during the course of the cell-adaptation process in MuNoV. These findings enhanced the understanding of processes related to laboratory adaptation of non-cultivable viruses, especially to those without relevant cell culture systems.

\section{AUTHOR CONTRIBUTIONS}

KaK and AN designed the study, performed the experiments, and wrote the manuscript. HT, AK, KuK, and RT-T performed the experiments. TK and KY performed bioinformatics analyses. All authors critically revised the manuscript and approved the final version.

\section{FUNDING}

This study was supported in part by funding from a commissioned project for the Research on Emerging and Re-emerging Infectious Diseases from the Japanese Ministry of Health, Labor, and Welfare, the Research Program on Emerging and Re-emerging Infectious Diseases from the Japan Agency for Medical Research and Development program, and the Japan Society for the Promotion of Science Grant-in-Aid for Scientific Research, KAKENHI.

\section{ACKNOWLEDGMENT}

We thank Dr. Yukinobu Tohya (Nihon University) for providing the MuNoV S7 strain.

\section{SUPPLEMENTARY MATERIAL}

The Supplementary Material for this article can be found online at: http://journal.frontiersin.org/article/10.3389/fmicb.2017. 01091/full\#supplementary-material 


\section{REFERENCES}

Acevedo, A., Brodsky, L., and Andino, R. (2014). Mutational and fitness landscapes of an RNA virus revealed through population sequencing. Nature 505, 686-690. doi: $10.1038 /$ nature 12861

Andino, R., and Domingo, E. (2015). Viral quasispecies. Virology 47, 46-51. doi: 10.1016/j.virol.2015.03.022

Arias, A., Urena, L., Thorne, L., Yunus, M. A., and Goodfellow, I. (2012). Reverse genetics mediated recovery of infectious murine norovirus. J. Vis. Exp. 64:e4145. doi: $10.3791 / 4145$

Baert, L., Wobus, C. E., Van Coillie, E., Thackray, L. B., Debevere, J., and Uyttendaele, M. (2008). Detection of murine norovirus 1 by using plaque assay, transfection assay, and real-time reverse transcription-PCR before and after heat exposure. Appl. Environ. Microbiol. 74, 543-546. doi: 10.1128/AEM.010 39-07

Bailey, D., Thackray, L. B., and Goodfellow, I. G. (2008). A single amino acid substitution in the murine norovirus capsid protein is sufficient for attenuation in vivo. J. Virol. 82, 7725-7728. doi: 10.1128/JVI.00237-08

Barron, E. L., Sosnovtsev, S. V., Bok, K., Prikhodko, V., Sandoval-Jaime, C., Rhodes, C. R., et al. (2011). Diversity of murine norovirus strains isolated from asymptomatic mice of different genetic backgrounds within a single U.S. research institute. PLoS ONE 6:e21435. doi: 10.1371/journal.pone.0021435

Duizer, E., Schwab, K. J., Neill, F. H., Atmar, R. L., Koopmans, M. P., and Estes, M. K. (2004). Laboratory efforts to cultivate noroviruses. J. Gen. Virol. 85(Pt 1), 79-87. doi: 10.1099/vir.0.19478-0

Ettayebi, K., Crawford, S. E., Murakami, K., Broughman, J. R., Karandikar, U., Tenge, V. R., et al. (2016). Replication of human noroviruses in stem cellderived human enteroids. Science 353, 1387-1393. doi: 10.1126/science.aaf5211

Fischer, A. E., Wu, S. K., Proescher, J. B., Rotem, A., Chang, C. B., Zhang, H., et al. (2015). A high-throughput drop microfluidic system for virus culture and analysis. J. Virol. Methods 213, 111-117. doi: 10.1016/j.jviromet.2014.12.003

Haga, K., Fujimoto, A., Takai-Todaka, R., Miki, M., Doan, Y. H., Murakami, K., et al. (2016). Functional receptor molecules CD300lf and CD300ld within the CD300 family enable murine noroviruses to infect cells. Proc. Natl. Acad. Sci. U.S.A. 113, E6248-E6255. doi: 10.1073/pnas.1605575113

Jones, M. K., Watanabe, M., Zhu, S., Graves, C. L., Keyes, L. R., Grau, K. R., et al. (2014). Enteric bacteria promote human and mouse norovirus infection of B cells. Science 346, 755-759. doi: 10.1126/science.1257147

Karst, S. M., Wobus, C. E., Lay, M., Davidson, J., and Virgin, H. W. T. (2003). STAT1-dependent innate immunity to a Norwalk-like virus. Science 299, 1575-1578. doi: 10.1126/science.1077905

Katayama, K., Murakami, K., Sharp, T. M., Guix, S., Oka, T., Takai-Todaka, R., et al. (2014). Plasmid-based human norovirus reverse genetics system produces reporter-tagged progeny virus containing infectious genomic RNA. Proc. Natl. Acad. Sci. U.S.A. 111, E4043-E4052. doi: 10.1073/pnas.1415096111

Kitajima, M., Oka, T., Tohya, Y., Katayama, H., Takeda, N., and Katayama, K. (2009). Development of a broadly reactive nested reverse transcription-PCR assay to detect murine noroviruses, and investigation of the prevalence of murine noroviruses in laboratory mice in Japan. Microbiol. Immunol. 53, 531-534. doi: 10.1111/j.1348-0421.2009.00152.x

Li, H., Handsaker, B., Wysoker, A., Fennell, T., Ruan, J., Homer, N., et al. (2009). The sequence alignment/map format and SAMtools. Bioinformatics 25, 2078-2079. doi: 10.1093/bioinformatics/btp352

Mauroy, A., Taminiau, B., Nezer, C., Ghurburrun, E., Baurain, D., Daube, G., et al. (2016). High-throughput sequencing analysis reveals the genetic diversity of different regions of the murine norovirus genome during in vitro replication. Arch. Virol. 162, 1019-1023. doi: 10.1007/s00705-016-3179-y

McFadden, N., Bailey, D., Carrara, G., Benson, A., Chaudhry, Y., Shortland, A., et al. (2011). Norovirus regulation of the innate immune response and apoptosis occurs via the product of the alternative open reading frame 4. PLoS Pathog. 7:e1002413. doi: 10.1371/journal.ppat.1002413

Minor, P. D. (2015). Live attenuated vaccines: historical successes and current challenges. Virology 47, 379-392. doi: 10.1016/j.virol.2015.03.032

Ojosnegros, S., Beerenwinkel, N., Antal, T., Nowak, M. A., Escarmis, C., and Domingo, E. (2010). Competition-colonization dynamics in an RNA virus. Proc. Natl. Acad. Sci. U.S.A. 107, 2108-2112. doi: 10.1073/pnas.090978 7107

Taube, S., Kolawole, A. O., Hohne, M., Wilkinson, J. E., Handley, S. A., Perry, J. W., et al. (2013). A mouse model for human norovirus. mBio 4:e450-13. doi: $10.1128 / \mathrm{mBio} .00450-13$

Thackray, L. B., Wobus, C. E., Chachu, K. A., Liu, B., Alegre, E. R., Henderson, K. S., et al. (2007). Murine noroviruses comprising a single genogroup exhibit biological diversity despite limited sequence divergence. J. Virol. 81, 10460-10473. doi: 10.1128/JVI.00783-07

Topfer, A., Zagordi, O., Prabhakaran, S., Roth, V., Halperin, E., and Beerenwinkel, N. (2013). Probabilistic inference of viral quasispecies subject to recombination. J. Comput. Biol. 20, 113-123. doi: 10.1089/cmb.2012.0232

Wobus, C. E., Karst, S. M., Thackray, L. B., Chang, K. O., Sosnovtsev, S. V., Belliot, G., et al. (2004). Replication of norovirus in cell culture reveals a tropism for dendritic cells and macrophages. PLoS Biol. 2:e432. doi: 10.1371/journal. pbio.0020432

Conflict of Interest Statement: The authors declare that the research was conducted in the absence of any commercial or financial relationships that could be construed as a potential conflict of interest.

Copyright (c) 2017 Kitamoto, Takai-Todaka, Kato, Kanamori, Takagi, Yoshida, Katayama and Nakanishi. This is an open-access article distributed under the terms of the Creative Commons Attribution License (CC BY). The use, distribution or reproduction in other forums is permitted, provided the original author(s) or licensor are credited and that the original publication in this journal is cited, in accordance with accepted academic practice. No use, distribution or reproduction is permitted which does not comply with these terms. 